LONGINES appartient depuis bientôt un siècle à l'élite des fabriques suisses d'horlogerie et prouve chaque jour ses traditions de qualité et de précision.

\section{La montre}

LONGINES jouit d'un rayonnement inégalé dans 150 pays, justifiant pleinement l'appellation des Anglo-Saxons...

THE WORLD'S MOST HONOURED WATCH

IL EST TEMPS... LONGINES

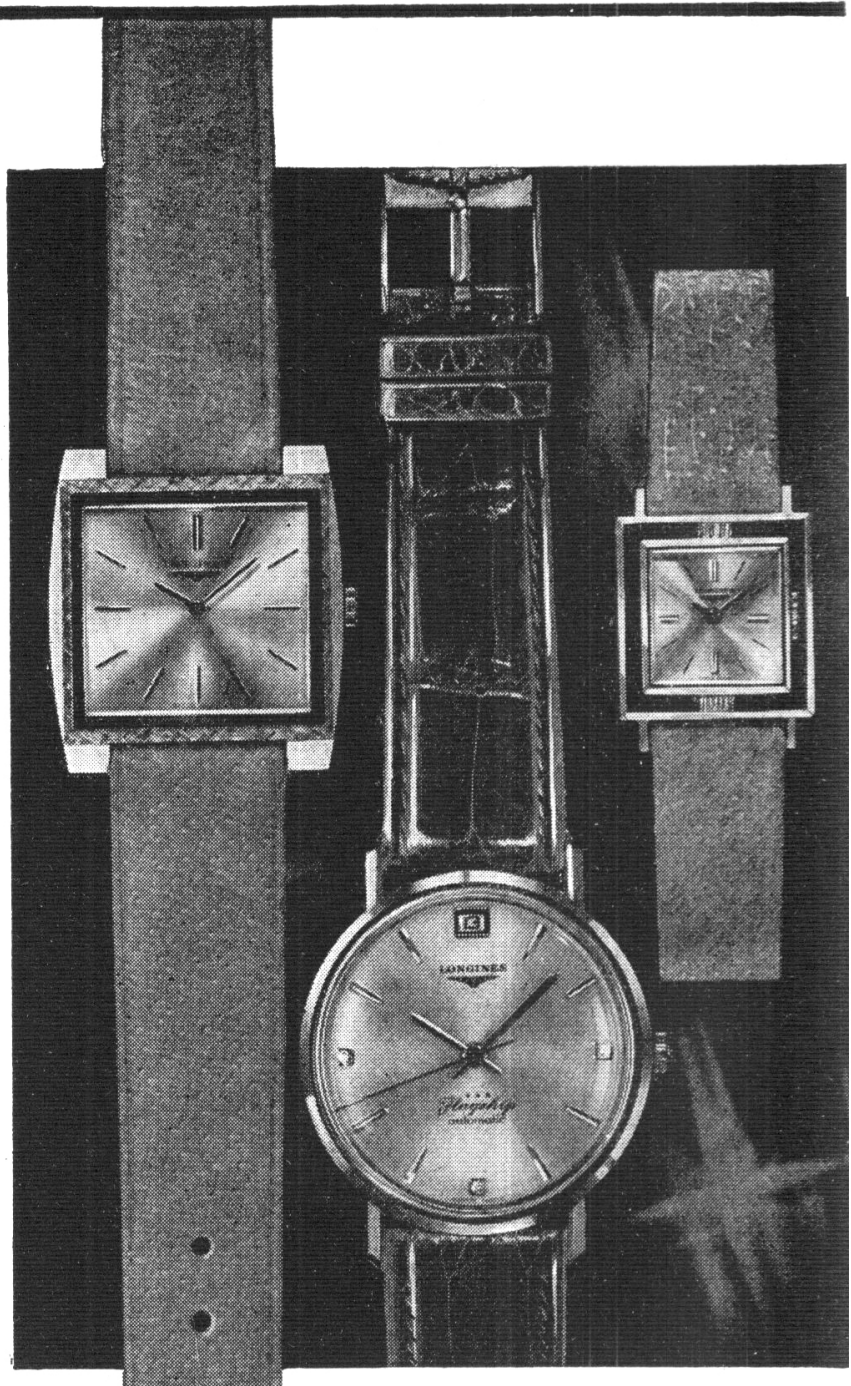

Réf. 7337 Boîtier forme tonneau; or $18 \mathrm{ct}$. Lunette lapidée, décor tuscanisé. Fr. 775. - .

Réf. 3508 Flagship $¥ \star \star$ Automatic calendrier, De Luxe; or 18 ct. cadran or massif avec brillants. Fr. 1090.- Réf. 7368 Boitier carré, avec cadre ajouré; or 18 ct. Fr. 765. - . 


\section{ADRESSES DES COMITÉS CENTRAUX}

AFGHANISTAN - Croissant-Rouge afghan, Kaboul.

AFRIQUE DU SUD (République) - Croix-Rouge sud-africaine, Cor. Kruis \& Market Streets, P.O.B. 8726, Johannesburg.

ALBANIE - Croix-Rouge albanaise, 35, Rruga e Barrikadavet, Tirana.

ALGÉRIE - Comité central du Croissant-Rouge algérien, 15 bis, boulevard Mohamed V, Alger.

ALLEMAGNE (République démocratique) - CroixRouge allemande dans la République démocratique allemande, 2, Kaitzerstrasse, Dresde $A l$.

ALlEMAGNE (République fédérale) - CroixRouge allemande dans la République fédérale d'Allemagne, 71, Friedrich-Ebert-Allee, 5300 Bonn 1, Postfach.

ARABIE SÉOUDITE - Croissant-Rouge de l'Arabie Séoudite, Riyad.

RÉPUBLIQUE ARABE UNIE - Croissant-Rouge de la République Arabe Unie, rue Ramsès, 34, Le Caire.

ARGENTINE - Croix-Rouge argentine, H. Yrigoyen 2068, Buenos - Aires.

AUSTRALIE - Croix-Rouge australienne, 122-128 Flinders Street, Melbourne, C.I.

AUTRICHE - Croix-Rouge autrichienne, 3, Gusshausstrasse, Postfach 39, Vienne IV.

BELGIQUE -- Croix-Rouge de Belgique, 98, chaussée de Vleurgat, Bruxelles 5.

BIRMANIE - Croix-Rouge de Birmanie, 42, Strand Road, Red Cross Building, Rangoon.

BOLIVIE - Croix-Rouge bolivienne, avenue SimonBolivar, 1515 (Casilla 741), La Paz.

BRÉSIL - Croix-Rouge brésilienne, Praça da Cruz Vermelha, 10-12, Rio de Janeiro.

BULGARIE - Croix-Rouge bulgare, 1 , boul. S. S. Biruzov, Sofia.

BURUNDI - Croix-Rouge du Burundi, B.P. 324 rue du Marché 3, Bujumbura.

CAMBODGE - Croix-Rouge cambodgienne, $17 \mathrm{R}$ Vithei Croix-Rouge cambodgienne, B.P. 94, Phnom-Penh.

CAMEROUN - Comité central de la Croix-Rouge camerounaise, rue Henri-Dunant, Boîte postale 631, Yaoundé.

CANADA - Croix-Rouge canadienne, 95, Wellesley Street East, Toronto, 5.

CEYLAN - Croix-Rouge de Ceylan, 106, Dharmapala Mawatte, Colombo VII.

CHILI - Croix-Rouge chilienne, Avenida Santa Maria, 0150 Correo, 15, Casilla 246.V., Santiago de Chile.

CHINE - Croix-Rouge chinoise, 22, Kanmien Hutung, Pékin, $E$.

COLOMBIE - Croix-Rouge colombienne, Carrera 7a, No. 34-65, Apartado Nacional 1110, Bogota D.E.

CONGO - Croix-Rouge du Congo, 41, av. Valcke, B.P.1712, Kinshasa.
CORÉE (République de) - Croix-Rouge de la République de Corée, 32-3 Ka Nam San Dong, Séoul.

CORÉE (République démocratique de) - CroixRouge de la République démocratique populaire de Corée, Pyongyang.

COSTA RICA - Croix-Rouge costaricienne, Calle $5^{\text {a }}$ Apartado 1025, San José.

COTE D'IVOIRE - Croix-Rouge de Côte d'Ivoire, B.P. 1244, Abidjan.

CUBA - Croix-Rouge cubaine, Calle Zuluerta 471, La Havane.

DAHOMEY - Société nationale de la Croix-Rouge du Dahomey, B.P. 1, Porto-Novo.

DANEMARK - Croix-Rouge danoise, Ny Vestergade 17, Copenhague $K$.

RÉPUBLIQUE DOMINICAINE - Croix-Rouge dominicaine, Calle Galvan, 24, Apartado 1293, Saint-Domingue.

ÉQUATEUR - Croix-Rouge équatorienne, Avenida Colombia y Elizalde, 118, Quito.

ESPAGNE - Croix-Rouge espagnole, Eduardo Dato, 16, Madrid, 10.

ÉTATS-UNIS - Croix-Rouge américaine, National Headquarters, $17_{\text {th }}$ and D. Streets, N.W., Washington $6, D . C$.

ÉTHIOPIE - Croix-Rouge éthiopienne, Red Cross Road No 1, P.O. Box 195, Addis-Abeba.

FINLANDE - Croix-Rouge de Finlande, Tehtaankatu, 1 A, P.O.B. 14168, Helsinki 14.

FRANCE - Croix-Rouge française, 17, rue QuentinBauchart, Paris $\left(8^{\mathrm{e}}\right)$.

GHANA - Croix-Rouge du Ghana, P.O. Box 835, Accra.

GRANDE-BRETAGNE - Croix-Rouge britannique, 14, Grosvenor Crescent, Londres, S.W.1.

GRÈCE - Croix-Rouge helléniqu^, Rue Lycavittou, 1, Athènes 135 .

GUATÉMALA - Croix-Rouge du Guatémala, $3^{a}$ Calle 8-40, Zona 1, Guatémala C.A.

HAÏTI - Croix-Rouge haïtienne, rue Férou, B.P. 1337, Port-alt-Prince.

HAUTE-VOLTA - Croix-Rouge voltalqque, B.P. 340, Ouagadougou.

HONDURAS - Croix-Rouge de Honduras, Calle Henri-Dunant № 516, Tegucigalpa.

HONGRIE - Croix-Rouge hongroise, Arany Janos utca, 31, Budapest $V$.

INDE - Croix-Rouge de l'Inde, Red Cross Road 1, La Nouvelle-Delhi, 1 .

INDONÉSIE - Croix-Rouge indonésienne, Tanah Abang Barat, 66, P.O. Box 2009, Djakarta.

IRAK - Croissant-Rouge de l'Irak, Al Mansour, Bagdad.

IRAN - Société du Lion-et-Soleil-Rouge de l'Iran, Avenue Ark, Téhéran. 


\section{ADRESSES DES COMITÉS CENTRAUX}

IRLANDE - Croix-Rouge irlandaise, 16, Merrion Square, Dublin 2.

ISLANDE - Croix-Rouge islandaise, øldugøtu 4, Post Box 872, Reykjavik.

ITALIE - Croix-Rouge italienne, 12, via Toscana, Rome.

JAMAİQUE - Croix-Rouge de la Jamalque, 76, Arnold Road, Kingston 5.

JAPON - Croix-Rouge du Japon, 5, Shiba Park, Minato-Ku, Tokio.

JORDANIE - Croissant-Rouge jordanien, P.O. Box 1337, Amman.

KÉNYA - Croix-Rouge du Kénya, St. John's Gate, B.O. Box 712, Nairobi.

LAOS - Croix-Rouge lao, B.P. 46, Vientiane.

LIBAN - Croix-Rouge libanaise, rue GénéralSpears, Beyrouth.

LIBÉRIA - Croix-Rouge du Libéria, National Headquaters, Corner of Tubman boulevard and $9 \mathrm{~h}$ Street Sinkor, P.O. Box 226, Monrovia.

LIBYE - Croissant-Rouge libyen, Berka Omar Mukhtar Street, P.O. Box 541, Benghazi.

LIECHTENSTEIN - Croix-Rouge du Liechtenstein, Vaduz.

LUXEMBOURG - Croix-Rouge luxembourgeoise, Parc de la Ville, C.P. 234, Luxembourg.

MADAGASCAR - Croix-Rouge de la République malgache, rue Clemenceau, B.P. 1168, Tananarive.

MALAISIE - Croix-Rouge de Malaisie, Jalan Belfield 519, Kuala Lumpur.

MALI - Croix-Rouge malienne, Route de Koulikora, B.P. 280, Bamako.

MAROC - Croissant-Rouge marocain, rue Calmette, B.P. 189, Rabat.

MEXIQUE - Croix-Rouge mexicaine, Avenida Ejercito Nacional No 1032, Mexico, $10, D F$.

MONACO - Croix-Rouge monégasque, bd de Suisse, 27, Monte-Carlo.

MONGOLIE (République populaire de) - CroixRouge de la République populaire de Mongolie, Central Post Office, Post Box 537, Oulan-Bator.

NÉPAL - Croix-Rouge du Népal, Tripureswore, P.B. 217, Kathmandu.

NICARAGUA - Croix-Rouge du Nicaragua, 12, Avenida Noroeste, Managua, D.N.

NIGER - Croix-Rouge du Niger, B.P. 386, Niamey.

NIGERIA - Croix-Rouge du Nigeria, Eko Akete Close, Ikoyi, Yaba, P.O. Box 764, Lagos.

NORVËGE - Croix-Rouge de Norvège, Parkveien, $33 \mathrm{~b}$, Oslo 3.

NOUVELLE-ZÉLANDE - Croix-Rouge néo-zélandaise, 61, Dixon Street, P.O.B. 6073, Wellington, C.2.

OUGANDA - Croix-Rouge de l'Ouganda, 17, Jinja Road P.O. Box 494, Kampala.

PAKISTAN - Croix-Rouge du Pakistan, Frere Street, Karachi 4.

PANAMA - Croix-Rouge de Panama, Apartado 668, Panama.

PARAGUAY - Croix-Rouge paraguayenne, calle André Barbero y Artigas 33, Asuncion.

PAYS-BAS - Croix-Rouge néerlandaise, 27, Prinsessegracht, La Haye.
PÉROU - Croix-Rouge péruvienne, Jiron Chancay 881, Lima.

PHILIPPINES - Croix-Rouge philippine, 860 , United Nations Avenue, P.O. Box 280, Manille.

POLOGNE - Croix-Rouge polonaise, Mokotowsica 14, Varsovie.

PORTUGAL - Croix-Rouge portugaise, Secrétariat général, Jardim 9 de Abril, 1-5, Lisbonne 3.

ROUMANIE - Croix-Rouge de la République Socialiste de Roumanie, Strada Biserica Amzei, 29, Bucarest.

SAINT-MARIN - Croix-Rouge de Saint-Marin, Palais gouvernemental, Saint-Marin.

SALVADOR, EL - Croix-Rouge du Salvador, $3^{\mathrm{a}}$ avenida Norte y $3^{\mathrm{a}}$, calle Poniente, 21, San Salvador.

SÉNÉGAL - Croix-Rouge sénégalaise, Bld. Fran: klin-Rooseveit, P.O.B. 299, Dakar.

SIERRA LEONE - Croix-Rouge de Sierra Leone, P.O. Box 427, 6, Liverpool Street, Freetown.

SOUDAN - Croissant-Rouge soudanais, P.O. Box 235, Khartoum.

SUĖDE - Croix-Rouge suédoise, Artillerigatan, 6, Stockholm 14.

SUISSE - Croix-Rouge suisse, Taubenstrasse, 8, Case postale 2699, 3001 Berne.

SYRIE - Croissant-Rouge syrien, 13, rue Abi-AlaAlmaari, Damas.

TANZANIE - Croix-Rouge de Tanzanie, Upanga Road, P.O.B. 1133, Dar-es-Salaam.

TCHÉCOSLOVAQUIE - Croix-Rouge tchécoslovaque, Thunovska, 18, Prague $I$.

THAIILANDE - Croix-Rouge thallandaise, King Chulalongkorn Memorial Hospital, Bangkok.

TOGO - Croix-Rouge togolaise, 19, avenue dos Alliés, B.P. 655, Lamé.

TRINITÉ-et-TQBAGO - Croix-Rouge de Trinit6 et-Tobago, 48, Pembroke Street, P.O. Box 357, Port of Spain.

TUNISIE - Croissant-Rouge tunisien, 19, rue d'Angleterre, Tunis.

TURQUIE - Société du Croissant-Rouge turc, Yenisehir, Ankara.

U.R.S.S. Alliance des Sociétěs de la Croix-Rouge et du Croissant-Rouge de l'U.R.S.S., Tcheremushkinskii proezd 5, Moscou, W 36.

URUGUAY - Croix-Rouge uruguayenne, Avenida 8 de Octubre 2990, Montevideo.

VENEZUELA - Croix-Rouge vénézuélienne, Avenida de Andrés Bello, No. 4, Apart. 3185, Caracas.

VIETNAM (République démocratique du) - CroixRouge de la République démocratique du Vietnam, 68, rue Bà-Trièu, Hanot.

VIETNAM (République du) - Croix-Roúge de la République du Vietnam, rue Hông Thâp Tu, No 201, Saigon.

YOUGOSLAVIE - Croix-Rouge yougoslave, Simina ulica broj, 19, Belgrade.

ZAMBIE - Croix-Rouge de Zambie, P.O. Box R. W. 1, Ridgeway, Lusaka. 